Universidad de Lima

Facultad de Psicología

Carrera de Psicología

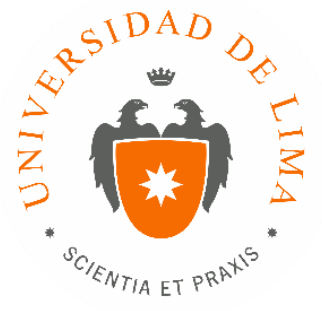

\title{
OPTIMIZACIÓN DEL PROCESO DE SELECCIÓN DE PERSONAL PARA PUESTOS OPERATIVOS
}

Trabajo de suficiencia profesional para optar el título profesional de Licenciado en Psicología

\section{Tania Lizett Vergara Rojas}

Código 20091211

Lima - Perú

Febrero de 2019 


\section{OPTIMIZACIÓN DEL PROCESO DE SELECCIÓN DE PERSONAL PARA PUESTOS OPERATIVOS}




\section{TABLA DE CONTENIDO}

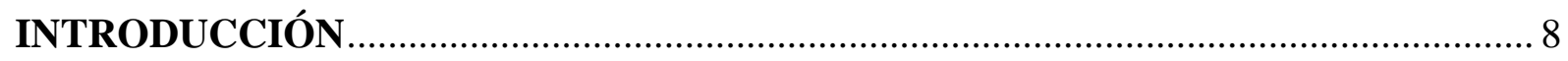

CAPÍTULO I: IDENTIFICACIÓN DEL PROBLEMA ............................................ 9

CAPÍTULO II: DESCRIPCIÓN DE LAS ACTIVIDADES REALIZADAS .................. 13

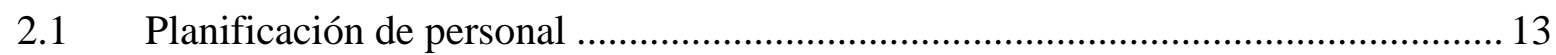

2.2 Flujograma del proceso de Reclutamiento y Selección ....................................... 14

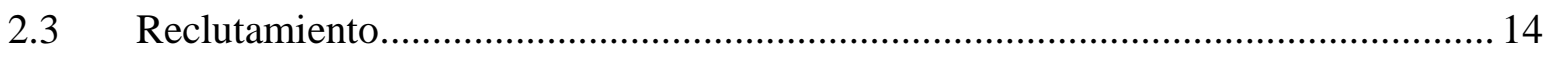

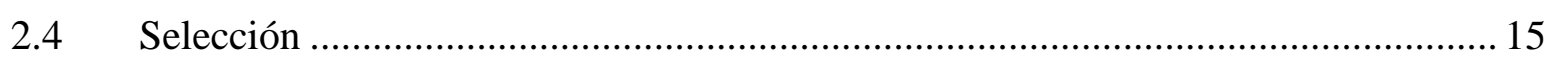

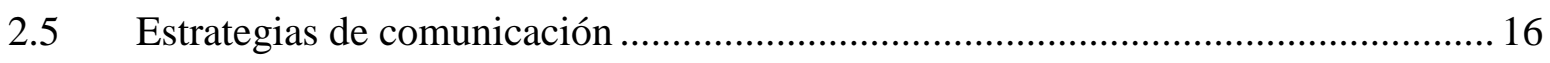

CAPÍTULO III: RESULTADOS DE LA INTERVENCIÓN ........................................ 17

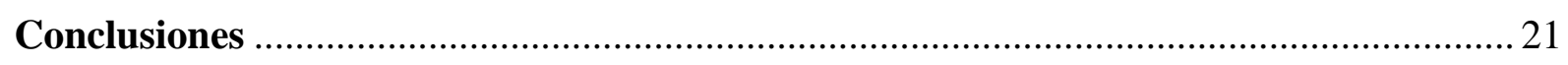

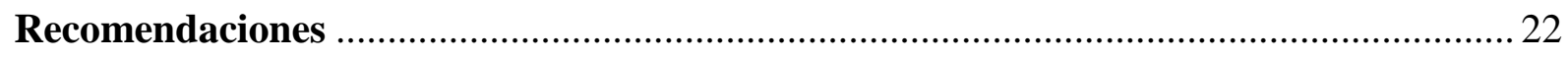

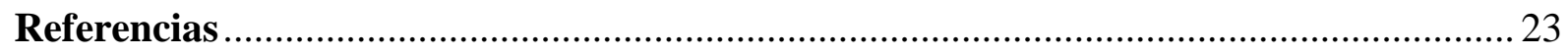

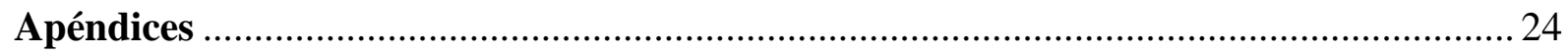




\section{ÍNDICE DE FIGURAS}

Figura 1.1: Porcentaje de satisfacción del proceso de selección de personal 2018 ................. 10

Figura 3.1: Porcentaje de satisfacción del proceso de selección de personal 2019. 19 


\section{ÍNDICE DE TABLAS}

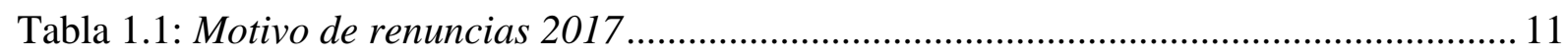

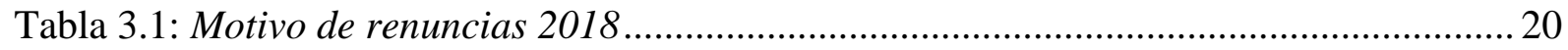




\section{ÍNDICE DE APÉNDICES}

Apéndice 1: Flujograma del Proceso de Selección De Personal ........................................... 25

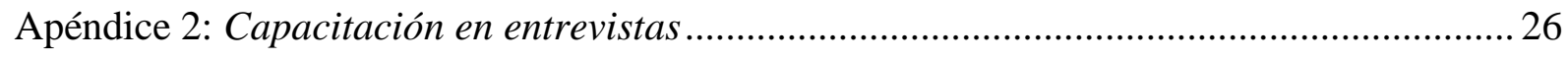

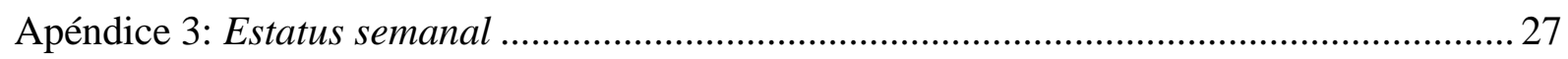




\section{INTRODUCCIÓN}

Toda organización tiene rotación, ya sea voluntaria o involuntaria, por lo que en algún momento necesitará cubrir el puesto vacante. Es ahí donde se requiere contar con un proceso de selección óptimo, que busque al candidato idóneo en el menor tiempo posible.

Para Chiavenatto (2014), el reclutamiento involucra diversas técnicas y procedimientos que se aplican para llegar a atraer a los candidatos más calificados que se incorporarán a la organización, mientras que, la selección de personal busca de entre todos los candidatos reclutados a aquellos que serán los más adecuados para cubrir el puesto con el objetivo de aumentar la eficacia de la organización.

Este trabajo se realiza a partir de la experiencia laboral de quien lo presenta, y tiene como finalidad dar a conocer uno de los tantos procesos que se gestionan en el área de Recursos Humanos, como es Reclutamiento y Selección de personal. Con ello se busca contribuir a quienes se desenvuelven dentro de esta área.

En el primer capítulo se presenta el planteamiento del problema y la información que fue obtenida, procesada y analizada a través de herramientas cuantitativas y cualitativas.

El segundo capítulo abarca todas las actividades que se implementaron para trabajar en planificación de personal, diseño del flujograma del proceso, reclutamiento, selección y estrategias de comunicación.

En relación con el tercer capítulo, se analizan los resultados obtenidos tras la aplicación de la propuesta de mejora. En ella se muestran los logros y su impacto en base al análisis de las cifras obtenidas.

Finalmente se exponen las conclusiones y recomendaciones frente al problema abordado. 


\section{CAPÍTULO I: IDENTIFICACIÓN DEL PROBLEMA}

Las organizaciones han ido cambiando a lo largo de los años y en la actualidad reconocen que para alcanzar sus objetivos es indispensable enfocarse en los procesos de gestión humana. Buscan diferenciarse en el mercado y a la vez son más exigentes.

El presente trabajo toma como escenario a la empresa HRC, perteneciente a un grupo de empresas peruanas. Se dedica a la fabricación y comercialización de redes, cabos e hilos para el sector de pesca y acuicultura. HRC, proyecta sus ventas y producción en base a las cifras del sector de pesca, las cuales fluctúan constantemente.

Cuenta con una sede central y una planta de producción. Está conformada por 383 colaboradores y está distribuida de la siguiente manera: 278 operarios, 19 técnicos, 6 practicantes y 80 administrativos.

A principios del 2018 se presentaron comentarios de inconformidad de parte del área de Operaciones con respecto al proceso de Selección de personal, argumentando la demora en cubrir los puestos, poca información al cliente interno, quejas por el comportamiento de los nuevos ingresos y percibían una alta rotación de personal.

Tomando en cuenta lo expuesto, se considera que dentro de los objetivos de cada organización está la rentabilidad, y para esto es necesario que todas las áreas contribuyan. Por su parte, el área de RR.HH es clave, pues como socio estratégico debe ir alineado al logro de los objetivos de negocio. Para ello, uno de los indicadores que toda organización debe de analizar es el índice de rotación, pues afecta de manera directa generando un gran impacto en términos de costos. De acuerdo con lo anterior, el presidente del directorio de la APERHU (Asociación Peruana de Recursos Humanos) declaró que el Perú cuenta con una de las tasas más altas de rotación de personal de América Latina (18\%), siendo del $5 \%$ al $10 \%$ en Latinoamérica, lo que genera sobrecostos a las organizaciones (Ipsos, 2014). Siendo más exactos, el sector manufacturero en 
el año 2015 fue uno de los sectores que presentó mayor rotación de personal con un porcentaje de $18.96 \%$ de rotación anual (Ministerio de Trabajo y Promoción del Empleo [MINTRA], 2015).

En el caso de HRC, en el 2017 obtuvo 58 \% de rotación anual, lo cual estaba muy por encima de las cifras mencionadas anteriormente. El mayor porcentaje lo obtuvieron los puestos operativos con un $60 \%$ de rotación anual, de los cuales el 54\% estuvieron menos de 3 meses. En relación con ello, uno de los procesos que impacta económicamente es el proceso de Reclutamiento y Selección de personal, ya que es indispensable contar con el candidato idóneo para el puesto dentro de una organización en el menor tiempo posible, pues esto permite que el cliente interno no se vea afectado por la falta de personal.

Se decidió recabar información para el diagnóstico de la situación actual. Para ello, se elaboró un cuestionario, instrumento cuantitativo, que fue aplicado a los Supervisores de producción. Esta herramienta consiste en un conjunto de preguntas que buscan medir alguna variable (Chasteauneuf, 2009 citado en Hernandez, Fernández y Baptista, 2014). El objetivo fue medir el nivel de satisfacción con el proceso de selección de personal, tomando en consideración los siguientes puntos: tiempo del proceso, perfil de los candidatos, comunicación con el encargado del proceso y satisfacción con el proceso.

\section{Porcentaje de Satisfacción del proceso de Selección de personal (2018)}

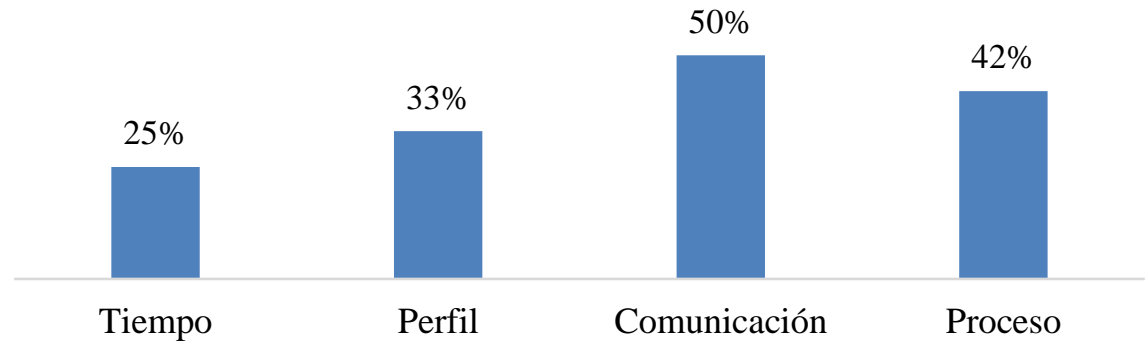

Figura 1.1. Resultados del cuestionario de satisfacción del proceso de selección de personal. Empresa HRC, Área de Recursos Humanos, 2018. 
En base a los resultados, se observó que la mayoría de los factores se encontraban por debajo del 50\% de satisfacción; siendo el menor, el tiempo de cobertura en los procesos.

Por otro lado, se decidió analizar las encuestas de salida para conocer el motivo por el cual los colaboradores decidían renunciar.

\section{Tabla 1.1}

Motivos de renuncia del personal operativo (2017)

\begin{tabular}{ll}
\hline Causas & $\mathrm{N}^{\circ}$ \\
\hline Problemas familiares & $28 \%$ \\
Mejor propuesta laboral & $5 \%$ \\
Estudios & $2 \%$ \\
Horario de trabajo & $20 \%$ \\
Remuneración & $15 \%$ \\
Dificultad con jefes & $10 \%$ \\
Capacitación & $12 \%$ \\
Falta de oportunidades de crecimiento & $8 \%$ \\
Total general & $100 \%$ \\
\hline
\end{tabular}

Nota: Empresa HRC, Área de Recursos Humanos, 2017.

Según las cifras, el mayor porcentaje de renuncias fue por problema familiar (28\%), seguido del horario de trabajo (20\%). Este segundo factor se contrastó con operaciones y nómina, donde efectivamente los colaboradores realizaban más horas extras de lo normal por la falta de personal.

Otra herramienta que se decidió utilizar fue la entrevista, técnica cualitativa. Para Vera, Lorente y Martínez (2012) las técnicas cuantitativas son insuficientes y en ocasiones es necesario un análisis más profundo de lo que se desea investigar. Para ello, se concretó una reunión con el Jefe de producción y los Supervisores donde se dio a conocer la inconformidad con los tiempos de cobertura; asimismo, resaltaron la poca comunicación durante el proceso y el desagrado por el comportamiento de los nuevos colaboradores. 
A lo largo de la reunión constantemente los Supervisores resaltaron que las renuncias o abandonos de trabajo se debían a que Selección no realizaba bien los filtros o no se les mencionaba las condiciones de trabajo, provocando falsas expectativas en los nuevos ingresos. Adicionalmente se llevó a cabo una reunión con el Jefe de Recursos Humanos, quién comentó que en el 2017 el tiempo promedio de cobertura para los puestos operativos fue de 12 días hábiles, estableciendo para el año 2018 un plazo de 10 días hábiles. Asimismo, resaltó que uno de los motivos por los que no se cubría en un menor tiempo era debido a la falta de planificación tanto de Recursos Humanos como de Operaciones. Por otro lado, resaltó la falta de un proceso de selección óptimo.

Al ahondar más a detalle sobre la gestión del proceso de Selección de personal en el 2017, se encontró que una de las fuentes de reclutamiento más utilizadas fueron los anuncios publicados en el exterior de la planta; sin embargo, había demoras en la etapa de Reclutamiento, ya que solo el $50 \%$ de los citados asistía el día de la evaluación. 


\section{CAPÍTULO II: DESCRIPCIÓN DE LAS ACTIVIDADES Y TAREAS REALIZADAS}

De acuerdo con los datos obtenidos se procedió a implementar estrategias que llevarían a optimizar el proceso de Reclutamiento y Selección de personal operativo.

\subsection{Planificación de personal}

Se identificó la demora en la cobertura de puestos vacantes ante una solicitud de requerimiento de personal. De acuerdo con Arbaiza (2016), la planificación permite anticiparse a las necesidades de la organización, cubriendo las vacantes a través de personal externo o brindando oportunidades de crecimiento.

Una de las estrategias que se decidió analizar fue el historial de los procesos de selección de los años 2016 y 2017. En base a ello, se determinó que en los meses de enero, mayo y setiembre había más solicitudes de requerimiento de personal. El primero, debido a que en el mes de diciembre los colaboradores solían renunciar luego de recibir su gratificación. En los meses de mayo y setiembre debido a que eran altas temporadas de pesca. Esto tuvo como objetivo poder planificar y generar acciones para lograr cubrir las vacantes en menor tiempo.

Se realizaron reuniones mensuales con el Jefe de producción y los Supervisores con el fin de conocer las necesidades o planes que producción tenía y anticiparse a ellas. Por ejemplo, una compra de maquinaria o aumento de producción por nuevos clientes. 


\subsection{Diseño del flujograma del proceso de Reclutamiento y Selección}

Debido a la falta de pasos importantes a la hora de llevar a cabo un proceso, se decidió elaborar el flujograma de reclutamiento y selección para puestos operativos, donde se establecieron los procedimientos que debía de cumplirse para seleccionar a los candidatos idóneos para cada puesto vacante (ver Apéndice 1).

\subsection{Reclutamiento}

En esta etapa se espera atraer a suficientes candidatos y captar a los más idóneos a ocupar los puestos vacantes. Para ello se debe tomar en cuenta el perfil del puesto antes de iniciar el proceso. Según Arbaiza (2016), el proceso de reclutamiento no solo se basa en el número de personas que se obtiene en una convocatoria sino también el tipo de personas y la posibilidad de aceptar o no la oferta laboral.

Tomando en cuenta esto, se procedió a diversificar las fuentes de reclutamiento.

Se adquirió una bolsa de trabajo por internet llamado Computrabajo y se generó una cuenta en Indeed. La finalidad fue realizar convocatorias abiertas con la intención de obtener candidatos en fechas y horarios establecidos para que los interesados puedan presentarse directamente y así optimizar el tiempo. Asimismo, se siguió utilizando el anuncio publicado en el exterior de la planta con el objetivo de no solo recibir hojas de vida sino también darse a conocer fechas y horarios fijos de convocatoria.

Por otro lado, se implementó un programa de referidos. La finalidad de este programa fue reducir el tiempo y los costos de reclutamiento. Se esperaba que los candidatos se adaptaran con mayor facilidad a la cultura organizacional, considerando que fueron informados por medio de un trabajador de la empresa. 
En este programa el colaborador podía referir a una persona ya sea conocido o familiar que considerara podía aplicar a una vacante. En caso el candidato referido era seleccionado y permanecía en la empresa mínimo 3 meses, el colaborador era acreedor de un vale de consumo de S/. 150. Con ello, se esperó crear una nueva fuente de reclutamiento que generaría mayor confianza.

Según Arbaiza (2016), contar con candidatos recomendados tiene ventaja, ya que podrán adaptarse rápidamente a la empresa, pues estarán familiarizados con ella debido a la relación con el colaborador.

Como parte de la etapa de reclutamiento, el día de las evaluaciones con Recursos Humanos se informaba a todos los asistentes sobre la empresa, condiciones de trabajo y las etapas del proceso. De esta manera se esperaba resolver las dudas que podían tener en ese momento y a su vez observar el comportamiento de los postulantes. Una vez informados, los candidatos tenían la opción de retirarse. Con esta actividad se esperaba optimizar tiempo y costos.

\subsection{Selección}

Puesto que se decidió no invertir tiempo en filtrar las hojas de vida y citar a los candidatos, se realizó el filtro curricular el día de la evaluación con aquellos que estaban de acuerdo con las condiciones de trabajo. El perfil de los puestos operativos contaba con requisitos básicos en relación con experiencia y estudios, por lo que no tomaba mucho tiempo este procedimiento.

Posteriormente, los candidatos llenaban una ficha de datos y realizaban las pruebas psicométricas utilizadas hasta el momento por la empresa. Las entrevistas se realizaron de manera individual con la finalidad de analizar más a profundidad e identificar a los más idóneos. Por tanto, "la entrevista de selección es un proceso de 
comunicación entre dos o más personas que interactúan y en el que una de las partes le interesa conocer lo mejor de la otra" (Chiavenato, 2009, p. 149).

Por otro lado, se decidió promover la participación de los Supervisores de producción en las entrevistas, como parte del proceso de selección de personal de puestos operativos. El objetivo era involucrarlos en el proceso y tener una idea más clara del perfil que deseaban incluir a su equipo de trabajo. En base a ello, para Chiavenato (2011) los jefes son quienes eligen a sus futuros colaboradores por lo que tienen una responsabilidad al elegir a los integrantes de su equipo. Asimismo, resultaba necesario un trabajo conjunto entre el área de Operaciones y el área de Selección para el cumplimiento de sus objetivos comunes. Para ello, se preparó una capacitación y entrenamiento en entrevistas efectivas señalando los puntos importantes que debían evaluar en el candidato y cómo desenvolverse en estas (ver Apéndice 2).

\subsection{Estrategias de comunicación}

La realización del cuestionario de satisfacción del cliente interno permitió identificar problemas de comunicación que percibía el Jefe de producción y los Supervisores por desconocer de los avances en los procesos de selección de puestos operativos. Para mejorar la comunicación, todos los viernes se informaba el estatus de los procesos, cantidad de puestos cubiertos y los aún pendientes por cubrir (ver Apéndice 3).

Asimismo, se establecieron reuniones mensuales para dar a conocer los indicadores de selección. Se mostraba el tiempo promedio de puestos cubiertos, ingresos según fuente de reclutamiento, procesos cubiertos en el mes y el motivo de solicitud de los requerimientos. 


\section{CAPÍTULO III: RESULTADOS DE LA INTERVENCIÓN}

En este capítulo se detallarán los resultados que se obtuvieron a lo largo de los meses de haber implementado una serie de actividades para optimizar el proceso de Reclutamiento y Selección de personal.

En relación con los tiempos de cobertura se logró, al cierre del año 2018, un tiempo promedio de 10 días hábiles para los puestos operativos, alcanzado el objetivo trazado por el área.

En cuanto al índice de rotación anual en puestos operativos en el 2018 fue de $51 \%$, cifra que disminuyó en comparación al año anterior; sin embargo, aún sigue siendo alta si se toma en cuenta los datos expuestos en el primer capítulo. Ello puede indicar que las actividades referentes al proceso de Selección de personal impactaron de manera positiva, pero aún podrían mejorarse las cifras.

Por otro lado, al hacer un análisis de las fuentes de reclutamiento, se identificó que el $56 \%$ del total de candidatos en el 2018 fueron reclutados a través del programa de referidos, de los cuales el $83 \%$ ingresó y el $68 \%$ superó los tres meses. De todos los requerimientos de perfil operativo solicitados en el 2018, el $43 \%$ fueron cubiertos a través de referidos. Estas cifras indican que el programa es una fuente importante de captación de talento que se puede seguir reforzando para obtener mejores resultados.

La elaboración del flujograma del proceso de Reclutamiento y Selección permitió establecer los pasos que debía seguir todo encargado del proceso para la búsqueda de un puesto operativo y no omitir aspectos importantes que a futuro traerían consecuencias económicas, contratación errónea, que perjudicarían al área solicitante.

Las entrevistas individuales permitieron identificar aspectos psicolaborales que posiblemente en una corta presentación serían difíciles de percibir. Si bien es una herramienta de evaluación para conocer al candidato, ello requiere de tiempo, y si no hay suficientes evaluadores puede 
perjudicar el proceso. En el caso de HRC, en los meses con mayores solicitudes de personal fue necesaria la participación de más integrantes del equipo. De acuerdo con esto, para GómezMejía, Balkin, y Cardy (2016), la selección implica tomar de decisión de contratar o no contratar a los candidatos. Para esto se requiere definir las características necesarias para el éxito del trabajo y valorar a cada candidato en función a sus características generarles basadas en el perfil del puesto.

Antes de implementar la segunda etapa de evaluación, se realizó la capacitación y entrenamiento en entrevistas a los Supervisores de producción, quienes en todo momento mostraron disposición para aprender (asistiendo puntualmente y participando activamente).

En Julio del 2018 se evidenció que el $95 \%$ de los candidatos aprobaban la segunda etapa del proceso. Al analizar ello, se logró identificar que el evaluador aprobaba a postulantes que incluso no estaba seguro si cumplían con el perfil, ya que no encontraba un sustento para rechazarlos. Ello se debe a la falta de experiencia en el proceso de entrevistas, por lo que a partir de la fecha se realizaron tres sesiones de acompañamiento para brindarles retroalimentación. Se debe considerar que dicha etapa es importante para el cliente interno, pues le permite conocer a los futuros integrantes de su equipo y, como líderes, aceptar que la necesidad de contar con candidatos idóneos para el puesto es un interés compartido.

En las primeras reuniones mensuales del 2018, marzo y abril, se percibió cierta resistencia de parte de los Supervisores de producción. Dicha actitud se evidenció al dar a conocer el índice de rotación mensual por área, señalando a Recursos Humanos como único responsable de los resultados obtenidos. Para llegar a un cambio de actitud, se hizo necesario continuas reuniones, logrando involucrar al Área de operaciones en el cumplimiento de los objetivos.

Uno de los beneficios observados a raíz de las reuniones mensuales fue poder anticipar las necesidades de personal en las distintas áreas de producción. Por ejemplo, se pudo planificar con anticipación el ingreso de 8 operarios para el mes de octubre, debido a una compra de 
maquinaria. Esto permitió contar con el personal antes de tiempo. Es así que, para GómezMejía, Balkin, y Cardy (2016), las organizaciones deben de emplear la planificación para asegurar cubrir sus necesidades de trabajo.

A inicios del 2018 se coordinó la implementación de herramientas cuantitativas para el diagnóstico de la problemática aplicando un cuestionario que permitiría medir el nivel de satisfacción del cliente interno en el proceso de Selección de personal para puestos operativos. En enero del 2019 se volvió a aplicar el mismo cuestionario con la intención de conocer sí las actividades implementadas tuvieron un impacto positivo.

\section{Figura 3.1}

Porcentaje de Satisfacción del proceso de Selección de personal

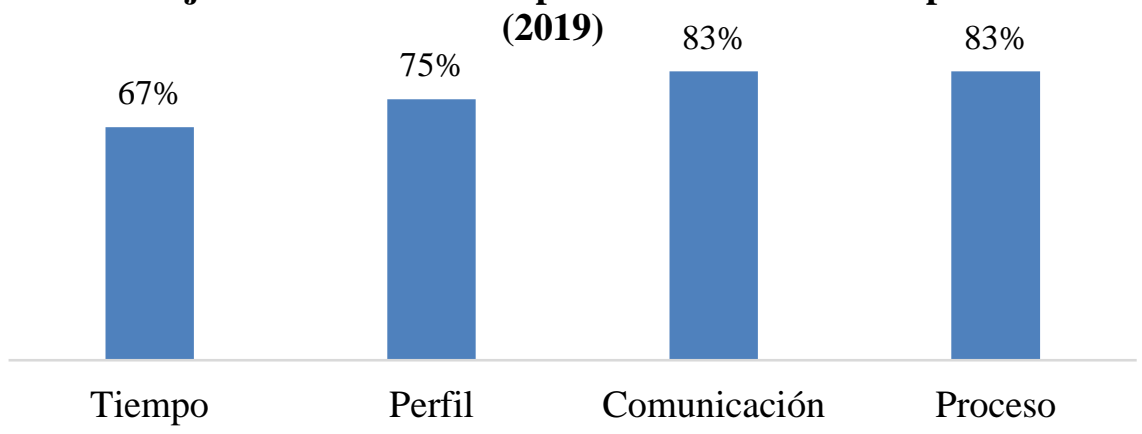

Figura 1: Resultados del cuestionario de satisfacción del proceso de selección de personal. Empresa HRC, Área de Recursos Humanos, 2019.

Según los resultados, se evidenció que todos los factores evaluados subieron, siendo la comunicación y el proceso los más altos con $83 \%$, mientras que el menor fue el tiempo de cobertura con $67 \%$. Si bien este último factor obtuvo un mejor resultado en comparación del 2018, se considera que el cliente interno en su mayoría siempre va a desear que el puesto se cubra en el menor tiempo posible o incluso de manera inmediata. 
Tabla 3.1

Motivos de renuncia del personal operativo 2018

\begin{tabular}{ll}
\hline Causas & $\mathrm{N}^{\circ}$ \\
\hline Problemas familiares & $20 \%$ \\
Mejor propuesta laboral & $8 \%$ \\
Estudios & $5 \%$ \\
Horario de trabajo & $11 \%$ \\
Remuneración & $13 \%$ \\
Dificultad con jefes & $17 \%$ \\
Capacitación & $11 \%$ \\
Falta de oportunidades de crecimiento & $15 \%$ \\
Total general & $100 \%$ \\
\hline
\end{tabular}

Nota: Empresa HRC, Área de Recursos Humanos, 2018.

En base a los resultados de las encuestas de salida 2018, los mayores porcentajes de renuncias fueron por problemas familiares en un $20 \%$, mismo factor obtenido en el análisis del 2017, dificultad con jefes en un $17 \%$ y la falta de oportunidades de crecimiento en un $15 \%$.

Tomando en cuenta dichos datos se consideró importante profundizar a la hora de entrevistar en el entorno familiar y su situación actual. Asimismo, respecto a los dos últimos factores resulta necesario obtener mayor información para realizar un plan de mejora en otros procesos importantes dentro de RR.HH, como Clima laboral y Desarrollo.

Es por ello que, el aporte del trabajo realizado fue de tipo aplicado, ya que se implementaron medidas que permitieron optimizar el proceso de Reclutamiento y Selección de puestos operativos. Por tanto, a través de los indicadores se demostró el logro de los objetivos. 


\section{CONCLUSIONES}

- Con la implementación del programa de referidos se logró cubrir el $43 \%$ de los puestos operativos solicitados en el año 2018, generando así una nueva fuente de reclutamiento.

- Se logró reducir los tiempos de cobertura en los procesos operativos de 12 días a 10 días hábiles, cumpliendo el objetivo anual.

- La optimización en el proceso de selección contribuyó a la reducción del índice de rotación anual de puestos operativos de $60 \%$ en el 2017 a $51 \%$ en el 2018.

- Se incrementó el nivel de Satisfacción del proceso de Selección de personal en todos los factores evaluados.

- Las entrevistas individuales permitieron conocer de manera más cercana al candidato e identificar a aquellos que cumplían el perfil de puesto requerido; sin embargo, al incrementarse considerablemente el número de candidatos se presentaron dificultades para la realización de un filtro adecuado, pues el tiempo de la entrevista por candidato reducía.

- Las encuestas de salida mostraron que las renuncias se deben a problemas familiares (20\%), dificultad en la relación con sus jefes $(17 \%)$ y falta de oportunidades de crecimiento $(15 \%)$.

- Inicialmente los Supervisores de producción mostraron resistencia a participar en el proceso de Selección; sin embargo, a través de las reuniones y la exposición de los resultados, mes a mes, se logró un mayor compromiso e involucramiento en el proceso. 


\section{RECOMENDACIONES}

A continuación, se detallan las recomendaciones:

- Para realizar un proceso de selección masivo es importante la descripción real y transparente del puesto. Sólo así se convocará a aquellos candidatos interesados.

- Se recomienda informar a los candidatos de las fases del proceso de selección para generar tranquilidad y confianza, motivándolos a brindar lo mejor de ellos en cada fase.

- Es importante establecer una comunicación cercana con los candidatos, impregnado la filosofía de la empresa y los valores de la compañía para crear la mejor experiencia de contratación.

- Tener siempre presente que el proceso de selección genera tensión en los candidatos. Por ello, se debe contemplar una entrevista amena y cordial en quienes se presentan a las convocatorias. Aquellos que no cumplan con el perfil establecido comunicárselo de manera asertiva.

- En el caso de HRC, se recomienda seguir fortaleciendo el programa de referidos, pues resultó ser una gran fuente de reclutamiento que obtuvo resultados positivos.

- Involucrar al cliente interno en el proceso de Selección de personal es fundamental. Ello permite acompañarlos en las primeras entrevistas, brindándose la retroalimentación adecuada al resaltar oportunidades de mejora. 


\section{REFERENCIAS}

Arbaiza, F. L. (2016). Dirección de recursos humanos: el factor humano (1 ${ }^{\mathrm{era}}$ ed.). Lima: Esan.

Chiavenato, I. (2011). Administración de recursos humanos: el capital humano de las organizaciones. México D.F.: McGraw-Hill.

Chiavenato, I. (2009). Gestión del talento humano (3 $3^{\text {era }}$ ed.). México D.F.: McGraw Hill.

Gómez-Mejía, L. R., Balkin, D., y Cardy, R. L. (2016). Gestión de recursos humanos (8va ed.). Madrid: Pearson Educación.

Hernández, S. R., Fernández, C. C., y Baptista, L. P. (2014). Metodología de la investigación. México: McGraw-Hill.

Ipsos Perú. (2014). Ante una alta rotación de colaboradores, aumente el valor de su propuesta. Recuperado de https://www.ipsos.com/sites/default/files/2017-

02/ante_una_alta_rotacion_de_colaboradores_aumente_el_valor_de_su_propuesta.pdf Ministerio de Trabajo [MINTRA]. (2015). Estadísticas Laborales: Variación de empleo. Recuperado de https://www.trabajo.gob.pe/mostrarContenido.php?id=93\&tip=9

Vera, M. (2012). Técnicas cualitativas en recursos humanos. Madrid: Síntesis 
APÉNDICES 


\section{APÉNDICE 1: FLUJOGRAMA DEL PROCESO DE SELECCIÓN DE PERSONAL}

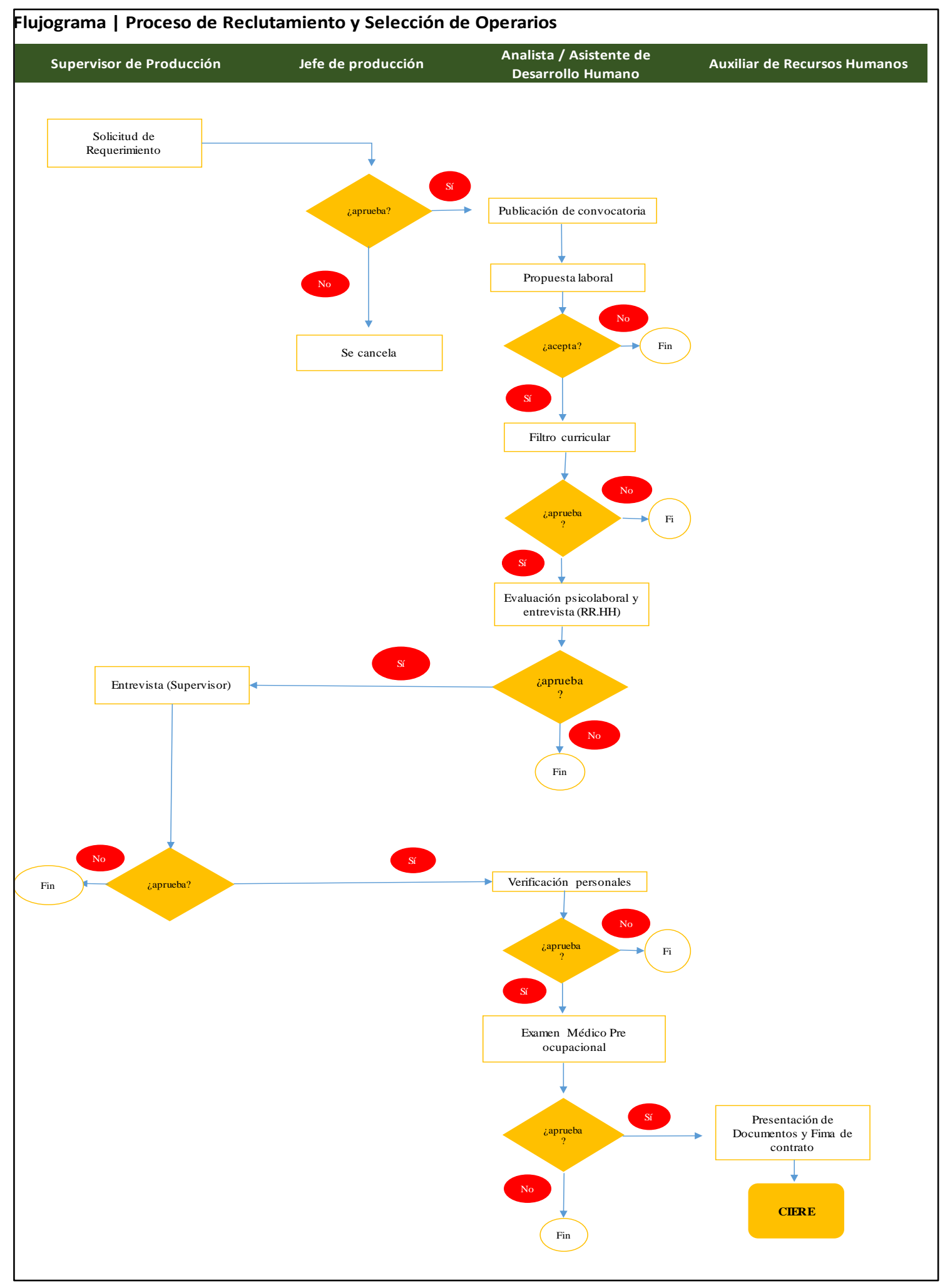




\section{APÉNDICE 2: CAPACITACIÓN EN ENTREVISTAS}

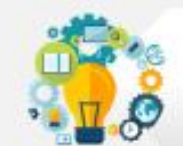

Dar herramientas para que los jefes realicen entrevistas efectivas de selección.

Facilitar la aplicación de un correcto filtro técnico en las entrevistas de selección.

Brindar material de consulta que facilite la realización de las entrevistas

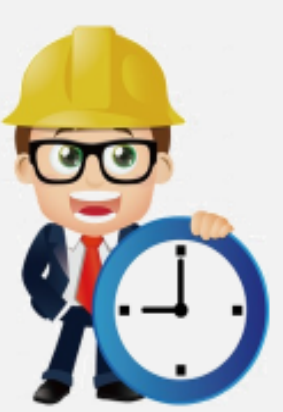

- Conocer al candidato.

- Probar sus actitudes personales.

- Verificar la personalidad y compatibilidad con el ambiente de trabajo

- Evaluar las competencias del candidato para el desarrollo eficaz del puesto.

- Transmitir una imagen adecuada de la empresa e informar al candidato sobre la empresa y el puesto

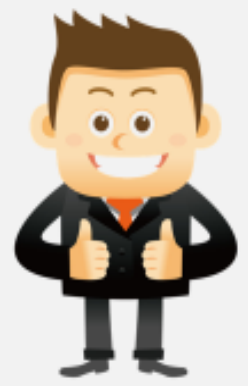

- Demostrar que nuestro perfil profesional y personal se adecua al del puesto ofertado.

- Demostrar su competencia laboral para el puesto, su interés en el mismo: sabe, quiere y puede desempeñar el puesto de trabajo.

- Causar una impresión positiva.

- Transmitir la información que nos solicitan.
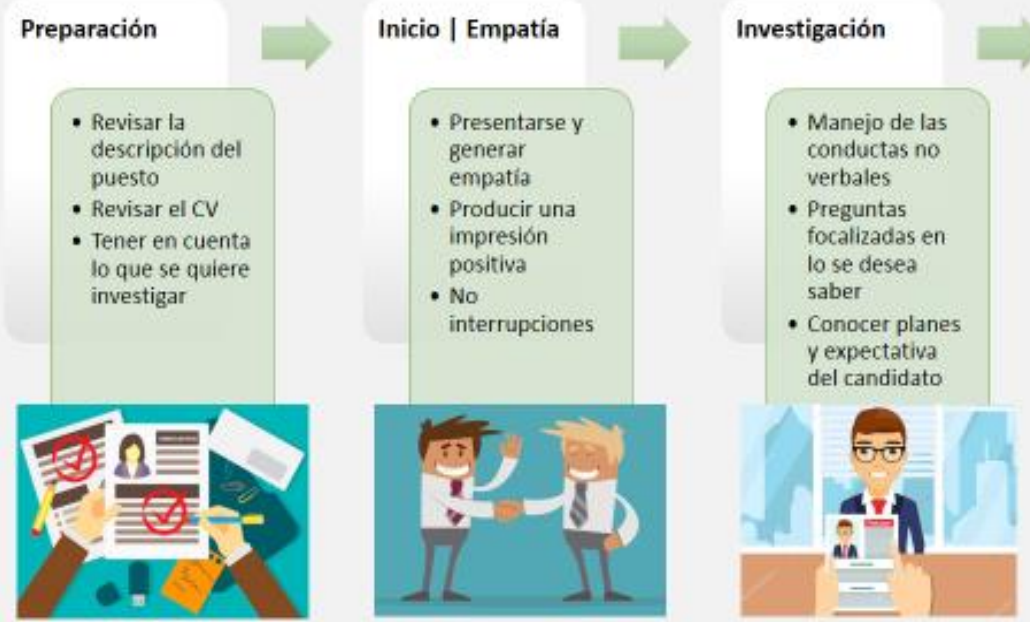

Cierre

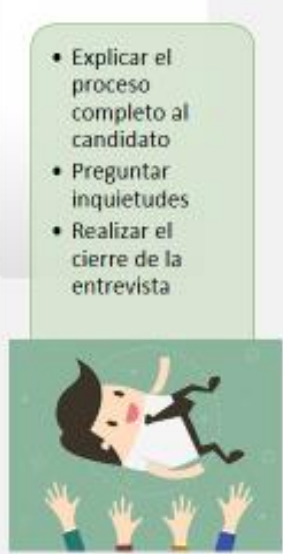




\section{APÉNDICE 3: ESTATUS SEMANAL}

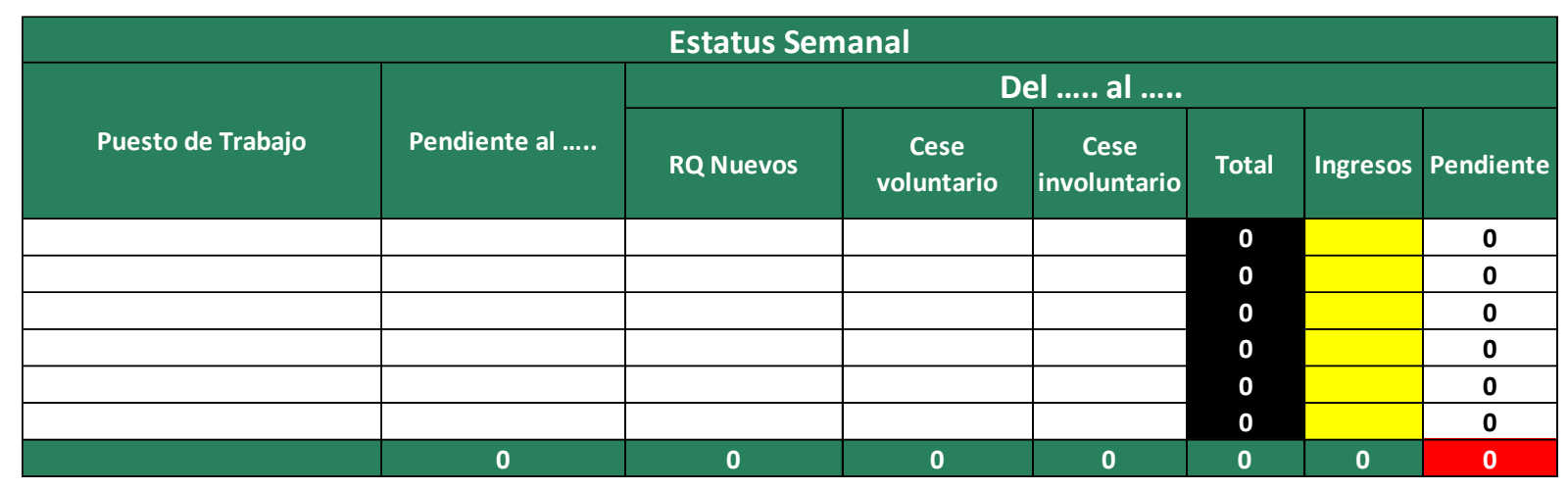

